\title{
A Matrix Metalloproteinase Inhibitor, ONO-4817, Suppresses the Development of Aortic Intimal Hyperplasia in Experimental Hyperlipidemic Rabbit
}

\author{
Yasuhiro OKamoto, ${ }^{1} \mathrm{MD}$, Kimio SATOMURA, ${ }^{1} \mathrm{MD}$, \\ Kazuhiro NAKAYAMA, ${ }^{1}$ MD, Nobukiyo TANAKA, ${ }^{1} \mathrm{MD}$, \\ Fumitaka OHSUZU, ${ }^{1}$ MD, Junko IMAKI, ${ }^{2}$ MD, Masahiko YosHIOKA, ${ }^{2}$ MD, \\ and Haruo NAKAMURA, ${ }^{3} \mathrm{MD}$
}

\begin{abstract}
SUMMARY
Inhibition of matrix metalloproteinases (MMPs) would be expected to suppress atherosclerotic neointimal proliferation and thus limit atheromatous plaque progression, but this has not yet been demonstrated morphologically in atherosclerotic intimal hyperplasia induced by cholesterol loading in experimental animals. We therefore investigated whether a broad-spectrum MMP inhibitor (MMPi), ONO-4817, could inhibit the development of intimal hyperplasia in male hyperlipidemic rabbits $(n=6)$ fed laboratory chow supplemented with $1 \%$ cholesterol for 2 months followed by a $1 \%$ cholesterol diet plus $100 \mathrm{mg} / \mathrm{kg}$ ONO-4817 for another month (Chol + ONO group). Control animals $(n=6)$ received no ONO-4817. When the aortas were studied both histologically and immunohistochemically, intimal hyperplasia was inhibited in Chol + ONO rabbits. The distribution of macrophages and MMP-12 in the hyperplastic tissue of the Chol + ONO rabbits was limited to the luminal side of the lesions. No such limitation in the distribution of macrophages and MMP-12 was observed in the control group. The distribution of smooth muscle cells in the hyperplastic tissue was not different between the Chol $+\mathrm{ONO}$ and control groups. However, the distribution of MMP-2 and MMP-12 was limited to the luminal side of lesions in the Chol + ONO group. This is the first reported evidence that an MMPi can suppress the development of intimal hyperplasia in hyperlipidemic rabbits. (Int Heart J 2007; 48: 369-378)
\end{abstract}

Key words: Atherosclerosis, Extracellular matrix, Matrix metalloproteinase, ONO-4817

MATRIX metalloproteinases (MMPs) have been shown to be expressed in vulnerable regions of atherosclerotic plaques ${ }^{1-3)}$ and are considered crucial in the progression and destabilization of atherosclerotic plaques. ${ }^{4,5)}$ Therefore, inhibiting the activity of these enzymes should limit the enlargement of these lesions and

From the ${ }^{1}$ First Department of Internal Medicine, ${ }^{2}$ First Department of Anatomy, National Defense Medical College, Saitama, and ${ }^{3}$ Mitsukoshi Health and Welfare Foundation, Tokyo, Japan.

Address for correspondence: Yasuhiro Okamoto, MD, First Department of Internal Medicine, National Defense Medical College, 2-3 Namiki, Tokorozawa, Saitama, Japan.

Received for publication October 25, 2006.

Revised and accepted February 16, 2007. 
enhance their stability. ${ }^{4-6)}$ Several studies with synthetic matrix metalloproteinase inhibitors (MMPi) have been conducted to test this hypothesis, but no convincing morphologic data have yet been obtained. ${ }^{7-9)}$

ONO-4817, a newly synthesized MMPi, is active as an MMPi after oral administration, and has a partially selective inhibitory spectrum (MMP-2, MMP8, MMP-9, MMP-12, and MMP-13 but not MMP-1 or MMP-7). ${ }^{10)} \mathrm{ONO}-4817$ is reported to inhibit metastasis in a variety of tumors ${ }^{11-15)}$ and also is reported to be able to inhibit neointimal formation by intimal injury in hypercholesterolemic hamster ${ }^{16)}$ and allograft vasculopathy. ${ }^{17)}$ The amount of MMP-2, MMP-9, and MMP-12 in neointimal lesions formed in hypercholesterolemic hamsters is reported to be decreased by ONO-4817. ${ }^{16}$

In this study, we present morphologic evidence that a broad-spectrum MMP inhibitor, ONO-4817, can suppress the development of atherosclerotic intimal hyperplasia induced by dietary hypercholesterolemia in experimental rabbits.

\section{Methods}

Animal preparation: Twelve 3-week-old Japanese white male rabbits were used. The rabbits were fed laboratory chow supplemented with $1 \%$ cholesterol to induce atherosclerotic intimal hyperplasia in the aorta. The Institutional Animal Care and Use Committee of the National Defense Medical College approved all of the animal protocols used in this study.

Lesion induction and treatment with ONO-4817: The twelve rabbits were fed laboratory chow supplemented with $1 \%$ cholesterol for 2 months. Among these rabbits, 6 were fed the same laboratory chow without any other interventions throughout the experimental period (control group). After 2 months of this $1 \%$ cholesterol diet, 6 other rabbits received $100 \mathrm{mg} / \mathrm{kg}$ of ONO-4817 orally in distilled water containing $0.5 \%$ sodium carboxymethyl cellulose for 1 month daily, while the $1 \%$ cholesterol feedings were continued (Chol + ONO group). After beginning ONO-4817 administration, body weight was recorded once a week to adjust the dose of ONO-4817 and to compare the body weights between the control and Chol + ONO groups. Statistical comparisons of the body weight between 2 groups were performed using Welch's $t$-test.

Serum lipid profiles of animals: Three representative rabbits in both groups were selected to monitor the serum lipid profiles (total cholesterol, HDL-cholesterol, triglycerides) during ONO-4817 administration. Statistical comparisons of each profile between the 2 groups were performed using Welch's $t$-test.

Histologic and immunohistochemical analyses: After 3 months of the treatment outlined above, the aortas were removed from the rabbits, fixed with $4 \%$ paraformaldehyde in phosphate-buffered saline at $\mathrm{pH} 7.2$, and embedded in par- 
affin. Serial 4- $\mu \mathrm{m}$ sections obtained from these tissues specimens were used for the histologic and immunohistochemical analyses. Routine hematoxylin and eosin ( $\mathrm{H}$ and $\mathrm{E}$ ) staining of transversely sectioned aortas was performed. After acquiring digital images of routinely $\mathrm{H}$ and $\mathrm{E}$ stained tissue by microscopy, the ratio of neointimal lesion to total cross sectional area of the aorta was analyzed using a computer image graphic analysis software program (VM32; Rise Corporation, Sendai, Japan). Five representative lesions located after the branching of the renal arteries were analyzed from 3 aortas (30 lesions total) and the results are expressed as the mean \pm SD. Statistical comparisons between the 2 groups were performed using Student's $t$-test.

The distribution of $\alpha$-actin detected by HHF-35 (Dako, USA, 1:2000 dilution) was used as a marker of vascular smooth muscle cells, while the distribution of macrophages was determined using RAM-11 (Dako, USA, 1:2000 dilution). The distributions of MMP-2 and MMP-9 were determined using monoclonal antibodies developed by Daiichi Fine Chemical, Japan (1:1000 dilution). The distribution of MMP-12 was determined using a polyclonal antibody developed by Biogenesis Ltd, UK (1:100 dilution). All sections were processed using a standard avidin-biotin complex method as previously described. ${ }^{18)}$

\section{RESUlts}

Macroscopic appearance of the aortas: Macroscopic appearance of a rabbit aorta is shown in Figure 1. Although the aortic intimal surfaces in the Chol + ONO
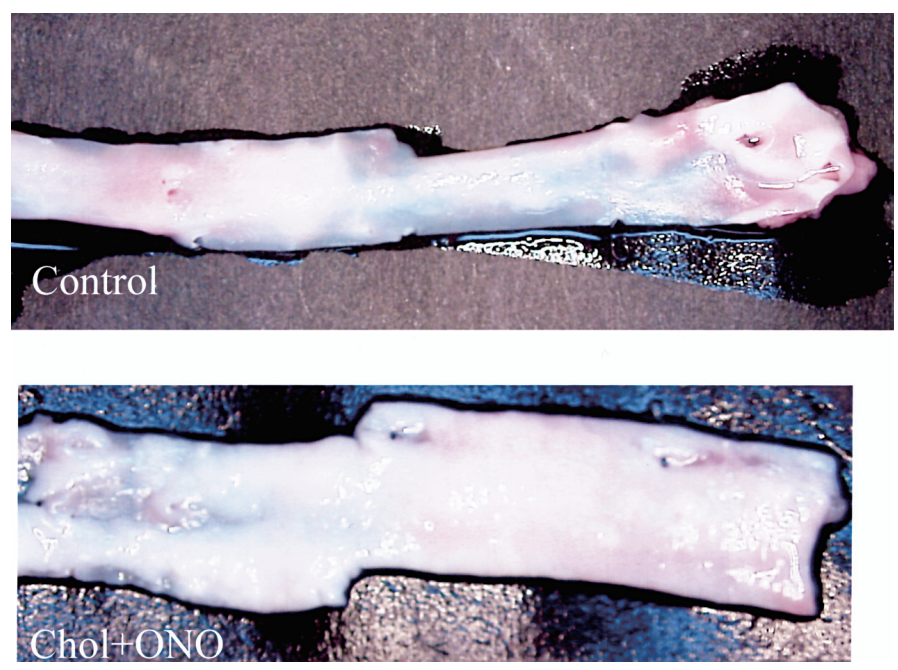

Figure 1. Macroscopic appearance of the aortas. 
group had a whitish-yellow appearance, no atherosclerotic plaques were obvious in the aortas of the Chol + ONO group, findings which are in contrast to those of

\section{$\mathrm{BW}(\mathrm{Kg})$}

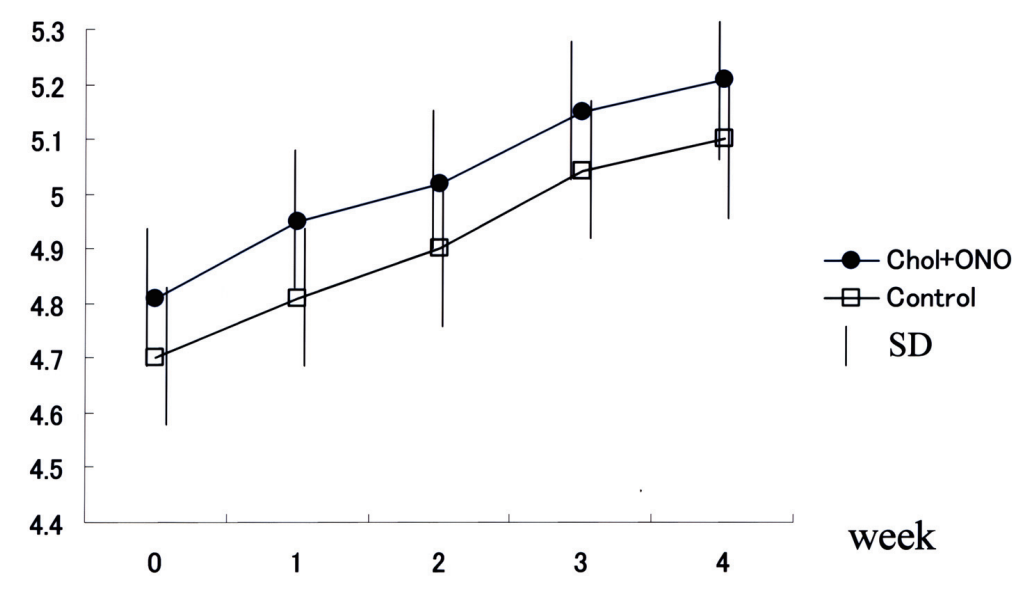

Figure 2. Body weight changes during the study. The mean $\pm \mathrm{SD}$ of the control and Chol $+\mathrm{ONO}$ groups during ONO-4817 administration. The mean body weight of the Chol + ONO group was greater than that of the control group during ONO-4817 administration.

\section{TC}

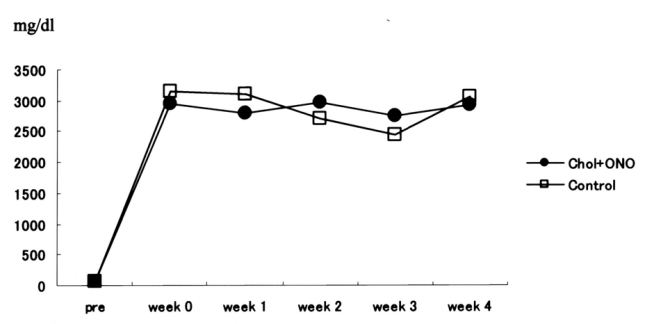

TG

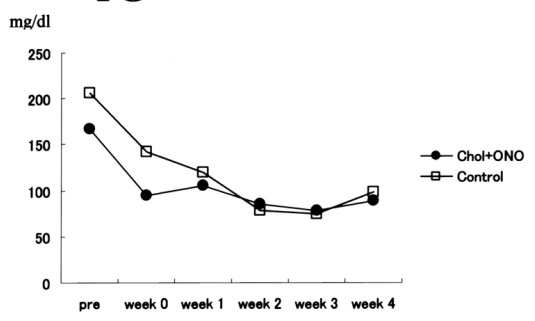

HDL-C

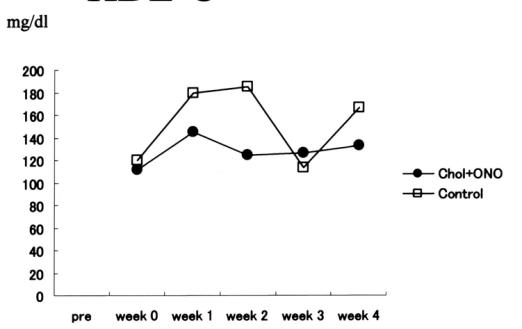

Figure 3. Lipid profile changes. Mean serum total cholesterol (TC), serum triglycerides (TG), and serum HDL-cholesterol (HDL-C) of the control and Chol + ONO groups before (pre) and during ONO-4817 administration (from week 0 to week 4). There were no statistically significant differences in any of the lipid profiles between the 2 groups. 
the control group.

Body weight change and lipid profiles of the animals: In both groups, the body weight increased during the study period. Although the average body weight of the Chol + ONO group was greater than that of the control group throughout the treatment period, no statistically significant difference was observed (Figure 2). There were no statistically significant differences in any of the serum lipid profiles between the 2 groups (Figure 3).

Histologic analysis: The histologic appearance of representative aortic crosssections is shown in Figure 4. This section was obtained distal to the branching of the renal arteries (Figure 4). The development of intimal hyperplasia was suppressed in the Chol + ONO group compared with the control group. The ratio of the neointimal lesion to the total cross sectional area (\%) was significantly greater in the control group $(19.85 \pm 9.44 \%)$ than in the Chol + ONO group $(6.52 \pm 1.32 \%$,

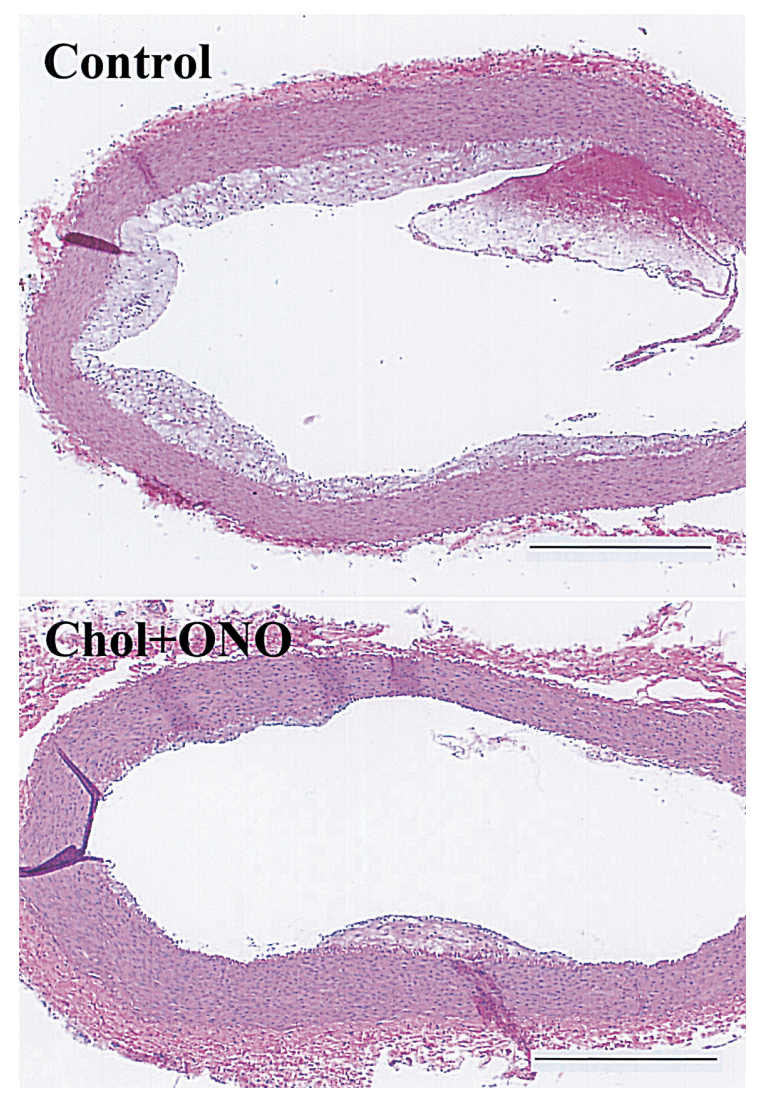

Figure 4. Histological analysis of cross sections of aortas from the control group (Control) and the Chol + ONO group (ONO). Scale bars $=50 \mu \mathrm{m}$. 
$P<0.001)$. In addition, the histological characteristics of the control group included a more advanced pattern of atherosclerosis than that in the Chol $+\mathrm{ONO}$
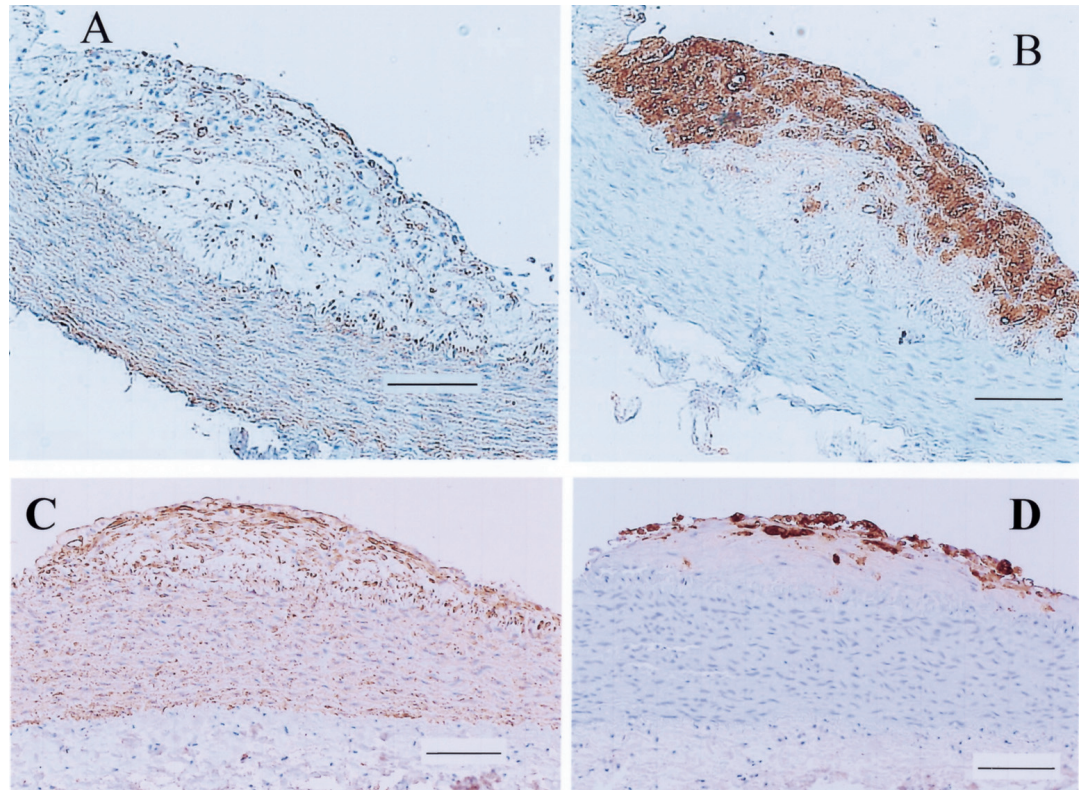

Figure 5. Immunohistochemical analysis of the distribution of smooth muscle cells ( $\alpha$-actin) and macrophages in lesions from both groups. The distribution of $\alpha$-actin in the control group (panel $\mathbf{A}$ ) did not differ from that in the Chol + ONO group (panel $\mathbf{C}$ ). Although macrophages were diffusely present in the region of atherosclerotic intimal hyperplasia in the control group (panel B), macrophages were not as common in the medial part of the region of intimal hyperplasia in the Chol + ONO group (panel D). Scale bars $=10 \mu \mathrm{m}$.
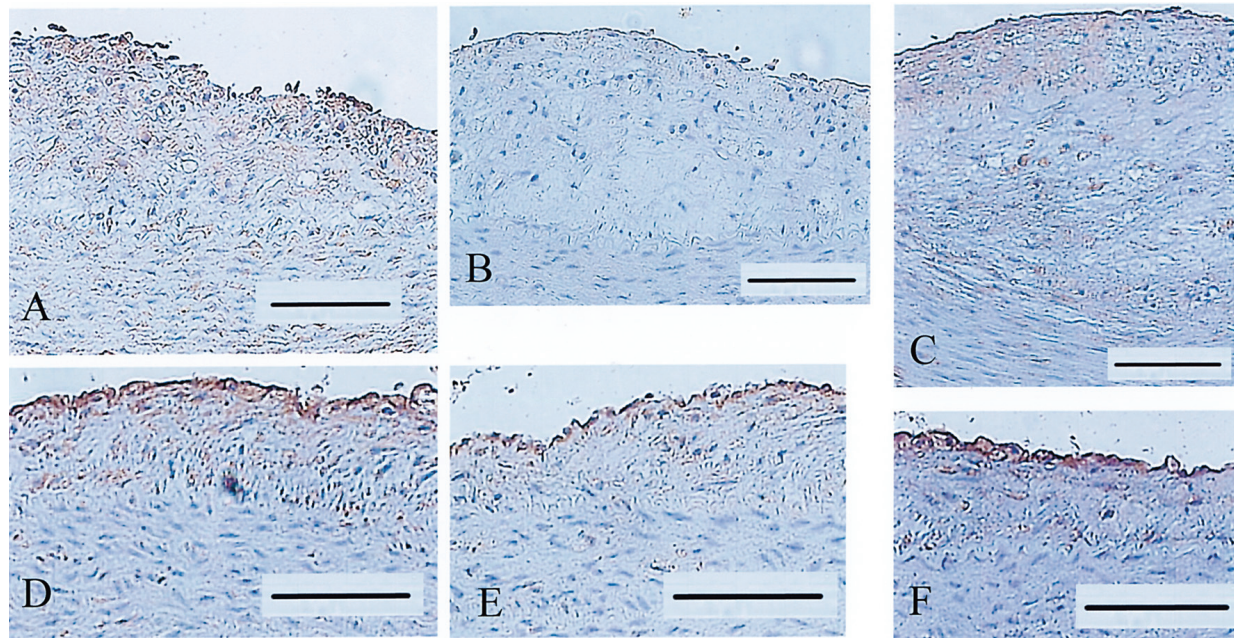

Figure 6. Immunohistochemical analysis of the distribution of MMP-2, MMP-9, and MMP-12 in lesions from both groups. MMP-2 was detected diffusely in the atherosclerotic neointima in the control group (panel A). The expression of this MMP was limited to the luminal aspect of the neointima in the Chol + ONO group (panel D). Distribution of MMP-9 did not differ between the control group (panel B) and the Chol + ONO group (panel E). MMP-12 was detected diffusely in the atherosclerotic neointima in the control group (panel $\mathbf{C}$ ). The distribution of this MMP was limited to the luminal aspect of the neointima in the Chol + ONO group (panel F). Scale bars $=10 \mu \mathrm{m}$. 

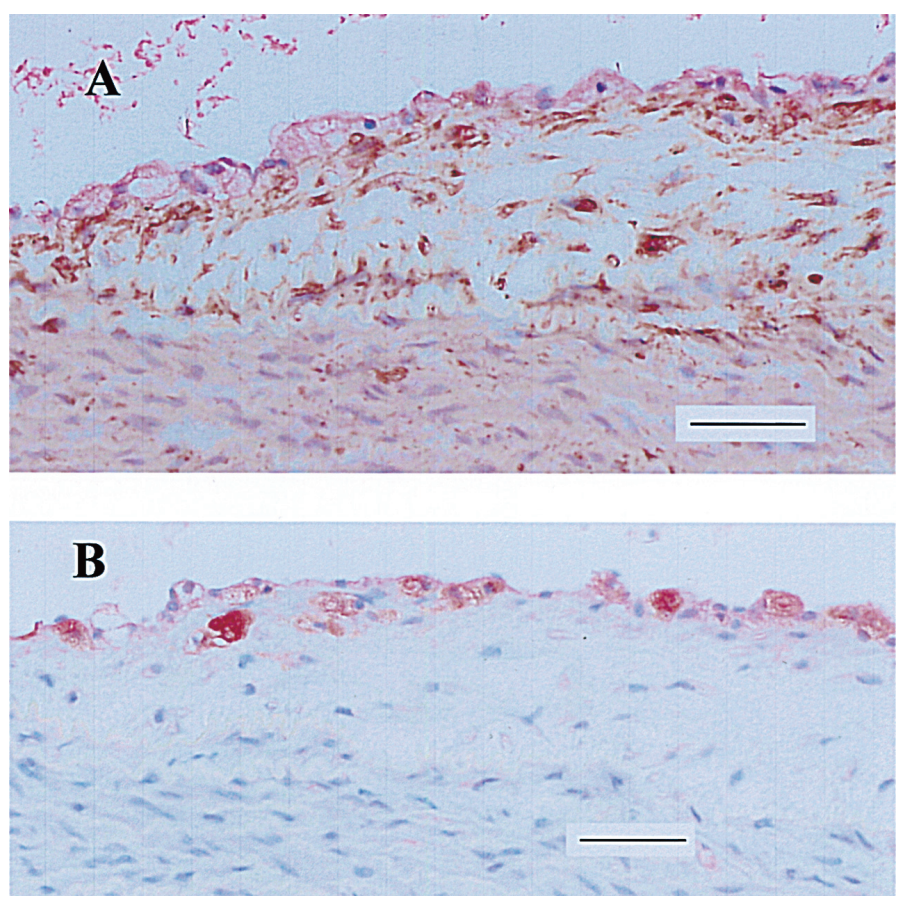

Figure 7. Immunohistochemical double staining for MMP-2 and smooth muscle cells and macrophages in the $\mathrm{Chol}+\mathrm{ONO}$ group.

Fucsin chromogen (red color) was used to detect MMP-2. Several $\alpha$-actin negative cells present in the luminal side of the lesion stained positive for MMP-2 (panel A). These cells were identified as macrophages (panel B). Scale bars $=5 \mu \mathrm{m}$.

group. In other words, on both the histological and macroscopic level, the atherosclerotic lesions in the Chol + ONO group were earlier lesions. This suggests that ONO-4817 can inhibit both the development and evolution of atherosclerotic lesions.

Immunohistochemical analysis: The distributions of $\alpha$-actin (reflecting smooth muscle cells) and of macrophages are shown in Figure 5. Although lesions in the Chol + ONO group were smaller than in the control group, the distribution pattern of $\alpha$-actin did not differ between the groups. However, the distribution of RAM11-positive cells (reflecting macrophages) was limited to the luminal aspect of the atherosclerotic neointima in the Chol + ONO group in contrast to the abundant population and the diffuse distribution of these cells in the intimal atherosclerotic lesions in the control group.

The distributions of MMP-2, MMP-9, and MMP-12 are summarized in Figure 6. Because MMP-2 and MMP-9 are reported to be responsible for the migration of smooth muscle cells ${ }^{19,20)}$ and MMP-12 is reported to be responsible for 
plaque instability, ${ }^{21)}$ we felt that it was important to determine whether ONO4817 could affect the distribution of these MMPs. Despite the fact that MMP-2 was detected diffusely in the atherosclerotic neointima in the control group (Figure 6A), expression of this MMP was limited in the luminal aspect of the neointima in the Chol + ONO group (Figure 6D). To identify the origin of MMP-2, immunohistochemical double staining for MMP-2 and both $\alpha$-actin and macrophages was performed (Figure 7). In the Chol + ONO group, cells that were identified as macrophages that did not stain for $\alpha$-actin were found in the luminal side of the lesion and stained positive for MMP-2.

The distribution of MMP-9 was not different between the control group (Figure 6B) and the Chol + ONO group (Figure 6E) .

MMP-12 was detected diffusely in the atherosclerotic neointima in the control group (Figure 6C). However, the distribution of this MMP was limited in the luminal aspect of the neointima in the Chol + ONO group (Figure 6F).

\section{DISCUSSION}

Previous reports on the effect of MMPis on atherogenesis have not demonstrated clear histologic effects in animals, including no change in the extent of atherosclerotic intimal hyperplasia. ${ }^{78)}$ We herein have shown that ONO-4817 could inhibit the atherosclerotic neointimal hyperplasia in experimental hyperlipidemic rabbits.

At first, it should be noted that body weight increased during the study in both groups of rabbits. This indicates that ONO-4817 does not affect dietary behavior. Even though there was no statistically significant difference between the body weights of the 2 groups, the higher body weight of the Chol + ONO group during ONO-4817 administration suggests that the inhibitory effect of ONO-4817 on intimal hyperplasia is not dependent on a dietary effect. In addition, we also confirmed that ONO-4817 did not affect the serum lipid profiles of the rabbits. These findings suggest that the inhibitory effect on neointimal hyperplasia by ONO-4817 was independent of the dietary effect and modification of the lipid profile.

The inhibitory effect of ONO-4817 on intimal hyperplasia in this study is thought to be caused by a difference in the distribution of macrophages within the lesion between the control group and the Chol + ONO group. Further studies are therefore needed to determine whether the inhibition of MMP-12 can block the migration of macrophages beneath the arterial intima in vivo.

The distribution of smooth muscle cells was not different between the control group and the Chol + ONO group. However, the distribution of MMP-2 was altered in the Chol + ONO group, although no difference was found in MMP-9 
Table. Difference in Inhibitory Activities of MMP Inhibitors

\begin{tabular}{ccccccccc}
\hline & MMP-1 & MMP-2 & MMP-3 & MMP-7 & MMP-8 & MMP-9 & MMP-12 & MMP-13 \\
\hline ONO-4817 & inactive & 0.73 & 42 & 2500 & 1.1 & 2.1 & 0.45 & 1.1 \\
RS-130830 & inactive & 0.3 & 9.5 & inactive & 0.9 & 0.4 & 0.7 & 0.5 \\
\hline
\end{tabular}

ONO-4817, values are mean Ki (nmol/L). RS-1130830, values are mean IC50 (nmol/L).

expression in either group. ONO-4817 has a strong inhibitory effect on MMP-2 and MMP-9. ${ }^{10)}$ Because these 2 MMPs are thought to be responsible for the migration of smooth muscle cells, blocking the migration of smooth muscle cells by the inhibition of these MMPs could inhibit the formation of atherosclerotic neointima. In fact, we found that the evolution and the prevalence of atherosclerotic intimal hyperplasia were inhibited by ONO-4817. In addition, based on the findings of in vitro studies, ONO-4817 did not inhibit either DNA synthesis in smooth muscle cells or the production of MMP-2 and MMP-9 by smooth muscle cells. ${ }^{16)}$

Quantitative analysis should therefore be performed to define the features of the lesions, especially the tissue distribution of collagens and other extracellular matrix components, as well as the lipid content of the lesions. Second, the efficacy of the drug in treating more advanced lesions should be investigated. Furthermore, the effect of the drug on monocyte/macrophage populations, both in the circulation and in the bone marrow, should be studied.

It should be emphasized that there have been no previous reports containing convincing morphologic data demonstrating that MMPis can affect the progression of atherosclerosis. ${ }^{7,8)}$ Because ONO-4817 may be the first MMPi that is able to suppress the development of intimal hyperplasia induced by intimal injury in hypercholesterolemic hamsters, ${ }^{16)}$ we have presented data on atherosclerotic intimal hyperplasia in dietary hypercholesteloremic rabbits in this report.

In previous studies ${ }^{7,8)}$ and a recent study by RS-130830, ${ }^{9}$ it was reported that intimal hyperplasia could not be attained by these drugs. The inhibitory activity of RS-130830 and ONO-4817 is summarized in Table. ${ }^{910)}$ The balance of inhibitory activity, not the strength of inhibitory activity, may explain the inhibition of intimal hyperplasia.

To establish the future therapeutic promise for MMPis in the treatment of atherosclerosis, further studies on MMPis in atherosclerosis are therefore eagerly awaited. 


\section{REFERENCES}

1. Galis ZS, Sukhova GK, Lark MW, Libby P. Increased expression of matrix metalloproteinases and matrix degrading activity in vulnerable regions of human atherosclerotic plaques. J Clin Invest 1994; 94: 2493-503.

2. Henney AM, Wakeley PR, Davies MJ, et al. Localization of stromelysin gene expression in atherosclerotic plaques by in situ hybridization. Proc Natl Acad Sci USA 1991; 88: 8154-8.

3. Brown DL, Hibbs MS, Kearney M, Loushin C, Isner JM. Identification of $92-\mathrm{kD}$ gelatinase in human coronary atherosclerotic lesions. Association of active enzyme synthesis with unstable angina. Circulation 1995; 91: 2125-31.

4. Ambrose JA, Martinez EE. A new paradigm of plaque stabilization. Circulation 2002; 105: 2000-4.

5. Celentano DC, Frishman WH. Matrix metalloproteinases and coronary artery disease: a novel therapeutic target. J Clin Pharmacol 1997; 37: 991-1000. (Review)

6. George SJ. Therapeutic potential of matrix metalloproteinase inhibitors in atherosclerosis. Expert Opin Investig Drugs 2000; 9: 993-1007. (Review)

7. Prescott MF, Sawyer WK, Von Linden-Reed JV, et al. Effect of matrix metalloproteinase inhibition on progression of atherosclerosis and aneurysm in LDL receptor-deficient mice overexpressing MMP-3, MMP-12, and MMP-13 and on restenosis in rats after balloon injury. Ann N Y Acad Sci 1999; 878: 179-90.

8. Cherr GS, Motew SJ, Travis JA, et al. Metalloproteinase inhibition and response to angioplasty and stenting in atherosclerotic primates. Arterioscler Thromb Vasc Biol 2002; 22: 161-6.

9. Jhonson JL, Fritsche-Danielson R, Behrendt M, et al. Effect of broad-spectrum matrix metalloproteinase inhibition on atherosclerotic plaque stability. Cardiovasc Res 2006; 71: 586-95.

10. Yamada A, Uegaki A, Nakamura T, Ogawa K. ONO-4817, an orally active matrix metalloproteinase inhibitor, prevents lipopolysaccharide-induced proteoglycan release from the joint cartilage in guinea pigs. Inflamm Res 2000; 49: 144-6.

11. Muraishi Y, Mitani N, Fuse H, Saiki I. Effect of a matrix metalloproteinase inhibitor (ONO-4817) on lung metastasis of murine renal cell carcinoma. Anticancer Res 2001; 21: 3845-52.

12. Sawada S, Murakami K, Yamaura T, Mitani N, Tsukada K, Saiki I. Therapeutic and analysis model of intrahepatic metastasis reflects clinical behavior of hepatocellular carcinoma. Jpn J Cancer Res 2002; 93: 190-7.

13. Shiraga M, Yano S, Yamamoto A, et al. Organ heterogeneity of host-derived matrix metalloproteinase expression and its involvement in multiple-organ metastasis by lung cancer cell lines. Cancer Res 2002; 62: 5967-73.

14. Yamashita T, Fujii M, Tomita T, et al. The inhibitory effect of matrix metalloptoteinase inhibitor ONO-4817 on lymph node metastasis in tongue carcinoma. Anticancer Res 2003; 23: 2297-302.

15. Yamamoto A, Yano S, Shiraga M, Ogawa H, Goto H, Miki T, Zhang H, Sone S. A third-generation matrix metalloproteinase (MMP) inhibitor (ONO-4817) combined with docetaxel suppresses progression of lung micrometastasis of MMP-expressing tumor cells in nude mice. Int J Cancer 2003; 103: 822-8.

16. Matsuno H, Ishisaki A, Nakajima K, Kozawa O. Effect of a synthetic matrix metalloproteinase inhibitor (ONO-4817) on neointima formation in hypercholesterolemic hamsters. J Cardiovas Pharmacol 2004; 44: 5765.

17. Hariya A, Takazawa K, Yamamoto T, Amano A. ONO-4817, a novel matrix metalloproteinase inhibitor, attenuates allograft vasculopathy in a rat cardiac transplant. J Heart Lung Transplant 2004; 23: 1163-9.

18. Okamoto Y, Satomura K, Ohusuzu F, Nakamura H, Takeuchi K, Yoshioka M. Expression of matrix metalloproteinase 3 in experimental atherosclerotic plaques. J Atheroscler Thromb 2001; 8: 50-4.

19. Johnson C, Galis ZS. Matrix metalloproteinases-2 and -9 differentially regulate smooth muscle cell migration and cell-mediated collagen organization. Arterioscler Thromb Vasc Biol 2004; 24: 54-60.

20. Galis ZS, Khatri JJ. Matrix metalloproteinases in vascular remodeling and atherogenesis: the good, the bad, and the ugly. Circ Res 2002; 90: 251-62.

21. Shogo K. Atherosclerotic instability and matrix metalloproteinases. J Jpn Coll Angiol 2003; 43: 699-705. 